\title{
ON SEMIALGEBRAIC POINTS OF DEFINABLE SETS
}

\author{
ARTUR PIȨKOSZ \\ Institute of Mathematics, Cracow University of Technology \\ Warszawska 24, 31-155 Kraków, Poland \\ E-mail:pupiekos@cyf-kr.edu.pl
}

\begin{abstract}
We prove that the semialgebraic, algebraic, and algebraic nonsingular points of a definable set in o-minimal structure with analytic cell decomposition are definable. Moreover, the operation of taking semialgebraic points is idempotent and the degree of complexity of semialgebraic points is bounded.
\end{abstract}

Given any structure (or a Tarski system) on $(\mathbf{R},+, \cdot)$ (see [2, p. 503] for the definition), let $A$ be a definable subset of $\mathbf{R}^{n}$.

We will use the following notation:

$$
\begin{aligned}
\partial A & =\bar{A} \backslash A, \\
\mathrm{Cl}(A) & =\left\{x \in \mathbf{R}^{n} \mid \text { the germ } A_{x} \text { is closed }\right\}(=\text { the exterior of } \partial A), \\
\mathrm{S}(A) & =\left\{x \in \mathbf{R}^{n} \mid \text { the germ } A_{x} \text { is semialgebraic }\right\}, \\
\operatorname{Alg}(A) & =\left\{x \in \mathbf{R}^{n} \mid \text { the germ } A_{x} \text { is algebraic }\right\}, \\
\operatorname{Nonsing}_{k}(A) & \left.=\left\{x \in \mathbf{R}^{n} \mid A_{x} \text { is algebraic, nonsingular }{ }^{1}\right) \text { of dimension } k\right\}, \\
\operatorname{Nash}(A) & =\left\{x \in \mathbf{R}^{n} \mid \operatorname{dim} A_{x}=\operatorname{dim} \text { Zar cl } A_{x}\right\} .
\end{aligned}
$$

PROPOSITION 1.

$$
\begin{aligned}
\mathrm{S}(A) & =\mathrm{S}(\bar{A}) \cap \mathrm{S}(\partial A) \\
\operatorname{Alg}(A) & =\mathrm{Alg}(\bar{A}) \cap \mathrm{Cl}(A) \\
\operatorname{Nonsing}_{k}(A) & =\operatorname{Nonsing}_{k}(\bar{A}) \cap \mathrm{Cl}(A)
\end{aligned}
$$

Moreover, $\mathrm{Cl}(A)$ is a definable set.

P r o of. The first equality follows from the theory of semialgebraic sets (see [1, p. 24]). The next two are obvious as well as the definability of $\mathrm{Cl}(A)$ is.

1991 Mathematics Subject Classification: Primary 14P10; Secondary 03C50, 32B20.

Received by the editors: January 29, 1998.

The paper is in final form and no version of it will be published elsewhere.

$\left.{ }^{1}\right)$ See $[1$, pp. 59-61] for the definition. 
Definition 1. We will call a germ $A_{a}$ trivial if it is equal to a germ of the empty set or of the whole space.

If $A_{a}$ is semialgebraic $(a \in \mathrm{S}(A))$ then we define the degree of complexity of this germ (or point) as follows. Given a description of the type

$$
A_{a}=\bigcup_{i=1}^{k} C_{i}=\bigcup_{i=1}^{k} \bigcap_{j=1}^{l_{i}} D_{i, j},
$$

where $D_{i, j}=\left\{\operatorname{sgn} f_{i, j}=T_{i, j}\right\}_{a}, T_{i, j} \in T=\{+, 0,-\}$ and $f_{i, j}$ are polynomials, the complexity of the description is defined as the sum of degrees of all nonzero polynomials appearing in this description (we count them with multiplicity).

The degree of complexity of the point a (or germ $A_{a}$ ) is the least complexity of all possible descriptions. We denote it by $\operatorname{deg}_{a} A$.

Remarks. A semialgebraic germ is trivial iff its degree of complexity is zero. If a germ is semialgebraic and nontrivial, then we can avoid polynomials of degree zero in its description.

Proposition 2. The set of semialgebraic points of $A$ of the degree of complexity bounded by a given number $M \in \mathbf{N}$ is definable.

Proof. For $M=0$, the set $\mathbf{R}^{n} \backslash$ bd $A$ is definable. For $M \geq 1$, apply the following equality

$$
\begin{aligned}
& \left\{x \in \mathrm{S}(A) \mid \operatorname{deg}_{x} A \leq M\right\}=\left\{x \in \mathbf{R}^{n} \mid\right. \\
& \exists f_{i, j} \in \mathcal{P}_{M}\left(\mathbf{R}^{n}\right) \quad \exists T_{i, j} \in\{+, 0,-\} \quad(i, j=1, \ldots, M) \quad \sum_{i, j=1}^{M} \operatorname{deg} f_{i, j} \leq M, \\
& \left.\exists \epsilon>0 \quad \forall y \in \mathbf{R}^{n} \quad(|x-y|<\epsilon) \Rightarrow\left(y \in A \Leftrightarrow \bigwedge_{i, j=1}^{M} \operatorname{sgn} f_{i, j}(y)=T_{i, j}\right)\right\},
\end{aligned}
$$

where $\mathcal{P}_{M}$ denotes the vector space (of finite dimension) of all polynomials of degree not exceeding $M$.

R e m a rk. One can get similar facts for points of definability in other structures if a given structure is the collection of all definable sets in some finite language.

In general, the semialgebraic points of a definable set are not of bounded degree of complexity (e.g. in the structure of all subsets, which is not o-minimal). It is so if the structure satisfies the following condition which is equivalent to the existence of analytic cell decompositions of definable sets (see the remark after 4.8 in [2] for one implication; the other has been noticed by W. Pawłucki and is easy to check) and implies o-minimality. (The author does not know an example of an o-minimal structure which does not admit analytic cell decompositions.)

Definition 2. We say that a structure $\mathcal{S}$ on $(\mathbf{R},+, \cdot)$ admits analytic stratifications if for every finite sequence of sets $A_{1}, \ldots, A_{k} \subset \mathbf{R}^{n}$ definable in (being members of) $\mathcal{S}$ there exists a finite analytic stratification of $\mathbf{R}^{n}$ into strata definable in $\mathcal{S}$ and compatible with all $A_{i}(i=1, \ldots, k)$.

(Recall that an analytic stratification is a locally finite decomposition into connected analytic submanifolds, called strata, satisfying the border condition: for strata $S, T$ if $S \cap \partial T \neq \emptyset$ then $S \subset \partial T$ and $\operatorname{dim} S<\operatorname{dim} T$.) 
Lemma 1 (see [3, p. 77]). Let $X \subset \mathbf{R}^{n}$.

1) If $X$ is semialgebraic, then there is an algebraic set $Y$ containing $X$ such that $\operatorname{dim} Y=\operatorname{dim} X$.

2) If $Y$ is an algebraic set, $X \subset Y$, then $X$ is semialgebraic iff both $(Y \cap \bar{X}) \backslash X$ and $X \backslash \operatorname{int}_{Y} X$ are semialgebraic.

Similar facts hold for germs.

THEOREM 1. If the structure admits analytic stratifications then $\mathrm{S}(A), \operatorname{Alg}(A)$, and $\operatorname{Nonsing}_{k}(A)$ for any $k=0, \ldots, n$ are definable.

Proof. By Proposition 1 we can assume that $A$ is closed. Now, we use induction on the dimension of $A$. For $\operatorname{dim} A=-\infty, 0$ the theorem is trivial. Let $\operatorname{dim} A>0$.

Let us take a finite analytic stratification for $A$ as in Definition 2. Take one stratum $S$ contained in $A$ and $x \in S$.

We have $A_{x}=S_{x} \cup \bigcup_{i=1}^{m} S_{i, x}$ where $S, S_{1}, \ldots, S_{m}$ are these strata of $A$ which have $S$ in their closures.

Assume that $x \in S \cap \mathrm{S}(A) \neq \emptyset$. Take the smallest algebraic set $B$ that contains $S, S_{1}, \ldots, S_{m}$. It follows that $\operatorname{dim} B=\operatorname{dim} A_{x}$ and $B_{x}$ is the smallest algebraic germ containing $A_{x}$. For each $y \in S$ we have $A_{y}=S_{y} \cup \bigcup_{i=1}^{m} S_{i, y} \subset B_{y}$. Thus $A_{y}$ is semialgebraic iff $\left(\operatorname{bd}_{B}(A \cap B)\right)_{y}$ is so. On the other hand, we know that $\operatorname{dim} \operatorname{bd}_{B}(A \cap B)<\operatorname{dim} B$ (see $[2,4.7])$, thus $\operatorname{dim} \operatorname{bd}_{B}(A \cap B)<\operatorname{dim} A$. Now, it is enough to use the induction hypothesis to establish definability of $\mathrm{S}(A) \cap S$ and, consequently, $\mathrm{S}(A)$.

If $x \in S \cap \operatorname{Alg}(A) \neq \emptyset$ then $S \cap \operatorname{Alg}(A)=\left\{y \in S \mid A_{y}=B_{y}\right\}=S \backslash \operatorname{bd}_{B}(A \cap B)$ is definable. Hence, $\operatorname{Alg}(A)$ is definable.

If $x \in S \cap$ Nonsing $_{k}(A) \neq \emptyset$, we have $A_{x}=B_{x}, B$ is irreducible, and $x \in \operatorname{Reg} B$, where $\operatorname{Reg} B$ is the set of nonsingular points $\left({ }^{2}\right)$ of $B$. Then $S \cap \operatorname{Nonsing}_{k}(A)=S \cap \operatorname{Reg} B \cap$ $\operatorname{ext}(B \backslash A)$ is definable and so is $\operatorname{Nonsing}_{k}(A)$.

THEOREM 2. If the structure admits analytic stratifications, then the degree of complexity of semialgebraic points on $A$ is bounded.

LEMMA 2. Boolean operations on sets and taking a connected component of a set preserve boundedness of the degree of complexity of semialgebraic germs, the degrees of complexity of the new germs depending only on the degrees of complexity of the initial germs.

Proof. It is easily checked that $\operatorname{deg}_{x}(A \cup B) \leq \operatorname{deg}_{x} A+\operatorname{deg}_{x} B$ and, by expressing an intersection of unions as a union of intersections, that $\operatorname{deg}_{x}\left(\mathbf{R}^{n} \backslash A\right) \leq \frac{1}{2}\left(2 \operatorname{deg}_{x} A\right)^{\operatorname{deg}_{x} A+1}$.

Now, using induction on $n$, we will prove the following statement:

For any natural $M, n$, there exist natural numbers $k(M, n), l(M, n)$ depending only on $M$ and $n$ such that for any semialgebraic set $D$ in $\mathbf{R}^{n}$ of degree of complexity $\leq M$, the number of connected components of $D$ is $\leq k(M, n)$ and each of the connected components is of degree of complexity $\leq l(M, n)$.

For $n=0$ or $M=0$ the statement is trivial. If $n>0$ then denote by $C$ a connected component of a semialgebraic set $D$ of degree of complexity not exceeding $M$. We follow the proof of Tarski-Seidenberg theorem. $D$ is described by not more than $M$ polynomials of degree not exceeding $M$. We divide $\mathbf{R}^{n-1}$ into bounded number of sets on which all

$\left({ }^{2}\right)$ Notation from [1]. 
polynomials have constant degree relative to the vertical axis. (From now on all polynomials are considered as families of polynomials on the vertical axis.) On each such set we take the product of all nonzero polynomials. This product is "prepared" by multiplication by all its nonzero derivatives. We divide our sets in $\mathbf{R}^{n-1}$ into smaller pieces on which the prepared polynomial has constant number of roots using generalized discriminants (which have bounded degree).

By the induction assumption, our pieces have bounded number of connected components each of them having bounded degree of complexity. The part of $\mathbf{R}^{n}$ over a connected component of a piece consists of not more than $2 M+1$ slices. The set $C$ is a union of some of such slices. There can be only a bounded number of such $C$ 's. Every such $C$ is a union of bounded number of slices having degree of complexity bounded. The estimating numbers depend only on $n$ and $M$. This ends the proof of the statement.

Now, facts about semialgebraic sets apply to semialgebraic germs.

Proof of Theorem 2. We can assume that $A$ is closed and use the induction on $\operatorname{dim} A$. For $\operatorname{dim} A=-\infty, 0$ the theorem is trivial. Assume that $\operatorname{dim} A>0$. Let us consider the situation from the proof of Theorem 1. If $x \in S \cap \mathrm{S}(A)$ then $A_{x}=$ $\left(\operatorname{bd}_{B}(A \cap B)\right)_{x} \cup\left(\operatorname{int}_{B}(A \cap B)\right)_{x}$. As we can take a substratification compatible with $B$, the germ ( $\left.\operatorname{int}_{B}(A \cap B)\right)_{x}$ is a union of a finite (and bounded) number of germs of connected components of $B \backslash \mathrm{bd}_{B}(A \cap B)$. By the induction assumption applied to $\operatorname{bd}_{B}(A \cap B)$ and by Lemma 2 , the degree of complexity of $A_{x}$ is bounded.

Definition 3. Let $E \subset \mathbf{R}^{n}$ and $x \in \mathbf{R}^{n}$. Then we define

$$
\begin{aligned}
& \partial_{x}^{1} E=(Z(E, x) \cap \bar{E}) \backslash E, \\
& \partial_{x}^{2} E=E \backslash \operatorname{int}_{Z(E, x)}(Z(E, x) \cap E),
\end{aligned}
$$

where $Z(E, x)$ is the (algebraic) Zariski closure of the germ $E_{x}$ (the smallest algebraic set whose germ contains $\left.E_{x}\right)$.

If $E$ is a union of some finite analytic stratification of $\mathbf{R}^{n}$ then $\partial_{x}^{1} E, \partial_{x}^{2} E$ do not depend on $x$ but only on the stratum to which $x$ belongs. Thus the family $\left\{\partial_{x}^{1} E, \partial_{x}^{2} E \mid x \in \mathbf{R}^{n}\right\}$ is finite.

For any finite sequence $\alpha=\left(\alpha_{1}, \alpha_{2}, \ldots, \alpha_{m}\right), m \in \mathbf{N}, \alpha_{i}=1,2$, we define $\partial_{x}^{\alpha} E$ inductively:

if $\alpha$ is the empty sequence (we write $\alpha=0$ ) then $\partial_{x}^{0} E=E$,

if $\alpha$ is the sequence $\alpha=\left(\alpha_{1}, \alpha_{2}, \ldots, \alpha_{m}\right), m \in \mathbf{N} \backslash\{0\}$, we set $\partial_{x}^{\alpha} E=\partial_{x}^{\alpha_{m}}\left(\partial_{x}^{\alpha^{\prime}} E\right)$, where $\alpha^{\prime}=\left(\alpha_{1}, \alpha_{2}, \ldots, \alpha_{m-1}\right)$. We define $|\alpha|=m$, the length of the sequence.

DEFINITION 4. Let $E$ be a union of strata of some analytic stratification $\mathcal{S}$ of $\mathbf{R}^{n}$. We say that a stratum $S \in \mathcal{S}$ is maximal for $E$ if $S \cap \bar{T} \neq \emptyset$ implies $T=S$ or $E \cap T=\emptyset$ for every $T \in \mathcal{S}$.

We define $\operatorname{Nash}^{\star}(E)=\left\{x \in \mathbf{R}^{n} \mid \forall k=1, \ldots, n, x \in \operatorname{Nash}\left(E^{k}\right)\right\}$ where $E^{k}$ is the equidimensional component of dimension $k$ of the set $E$.

THEOREM 3. If the structure admits analytic stratifications then $\mathrm{S}(\mathrm{S}(A))=\mathrm{S}(A)$.

Lemma 3. Let $A \subset \mathbf{R}^{n}$ and $x \in \mathbf{R}^{n}$. The following conditions are equivalent:

a) $x \in \mathrm{S}(A)$,

b) $x \in \bigcap_{|\alpha| \leq n} \operatorname{Nash}\left(\partial_{x}^{\alpha} A\right)$, 
c) $x \in \bigcap_{|\alpha| \leq n} \operatorname{Nash}^{\star}\left(\partial_{x}^{\alpha} A\right)$.

If $\mathcal{S}$ is an analytic stratification compatible with all $\partial_{x}^{\alpha} A,|\alpha| \leq n$ then each of the above conditions is equivalent to

d) $x \notin \overline{\mathcal{M}}_{A, x}$,

where $\mathcal{M}_{A, x}$ is the union of all strata maximal for some $\partial_{x}^{\alpha} A$ which are non-Nash manifolds.

Proof. Notice that $x \in \mathrm{S}(A)$ iff $x \in \operatorname{Nash}(A), x \in \mathrm{S}\left(\partial_{x}^{1} A\right)$, and $x \in \mathrm{S}\left(\partial_{x}^{2} A\right)$. Using the inclusions $\mathrm{S}(A) \subset \operatorname{Nash}^{\star}(A) \subset \operatorname{Nash}(A)$, we obtain

$$
\mathrm{S}(A)=\bigcap_{|\alpha| \leq n} \mathrm{~S}\left(\partial_{x}^{\alpha} A\right) \subset \bigcap_{|\alpha| \leq n} \operatorname{Nash}^{\star}\left(\partial_{x}^{\alpha} A\right) \subset \bigcap_{|\alpha| \leq n} \operatorname{Nash}\left(\partial_{x}^{\alpha} A\right) \subset \mathrm{S}(A)
$$

which proves that a), b), and c) are equivalent.

If the additional assumption is satisfied then $x \in \operatorname{ext} \mathcal{M}_{A, x}$ is equivalent to

$\forall|\alpha| \leq n \forall S$ maximal stratum of $\partial_{x}^{\alpha} A \quad(x \in \bar{S}) \Rightarrow(S$ is a Nash manifold $)$

which means that $x \in \bigcap_{|\alpha| \leq n} \operatorname{Nash}^{\star}\left(\partial_{x}^{\alpha} A\right)$. Thus c) is equivalent to d).

Proof of Theorem 3 . We can take a stratification $\mathcal{S}$ satisfying the assumption of d) in Lemma 3. Hence, the set of nonsemialgebraic points, described in d) of this lemma, is stable under iteration by its form.

Corollary. The set $\mathrm{N}(A)=\mathbf{R}^{n} \backslash \mathrm{S}(A)$ of nonsemialgebraic points of $A$ is closed, nowhere dense, definable and its dimension is never 0 or $n$. All its points are nonsemialgebraic.

Acknowledgements. The author wants to thank Ta Lê Loi, Krzysztof Kurdyka, Janusz Gwoździewicz, Andrzej Lenarcik and Wiesław Pawłucki for their valuable remarks, and The Fields Institute for invitation and supporting his stay in Toronto, where definability of semialgebraic points was proved.

\section{References}

[1] J. Bochnak, M. Coste, M.-F. Roy, Géométrie algébrique réelle, Ergeb. Math. Grenzgeb. (3) 12, Springer, Berlin, 1987.

[2] L. van den Dries, C. Miller, Geometric categories and o-minimal structures, Duke Math. J. 84 (1996), 497-540.

[3] S. Łojasiewicz, Ensembles semi-analytiques, Inst. de Hautes Études Scientifiques, Buressur-Yvette, 1965. 